Sociologie et sociétés

\title{
Travail, rapports sociaux, éducation en Europe
}

Esquisse historique de la chute de l'Empire à

l'Entre-deux-guerres

Work, Social Relations and Education in Europe

An Historical Outline from the Fall of the Empire to the Period between the Two World Wars

\section{Viviane ISAMBERT-JAMATI}

Volume 12, numéro 1, avril 1980

Éducation, économie et politique

URI : https://id.erudit.org/iderudit/001361ar

DOI : https://doi.org/10.7202/001361ar

Aller au sommaire du numéro

\section{Éditeur(s)}

Les Presses de l'Université de Montréal

\section{ISSN}

0038-030X (imprimé)

1492-1375 (numérique)

Découvrir la revue

Citer cet article

ISAMBERT-JAMATI, V. (1980). Travail, rapports sociaux, éducation en Europe : esquisse historique de la chute de l'Empire à l'Entre-deux-guerres. Sociologie et sociétés, 12(1), 9-22. https://doi.org/10.7202/001361ar

\section{Résumé de l'article}

Selon l'auteur, les transformations passées du système scolaire sont trop souvent ignorées des sociologues. Se basant sur des recherches récentes d'histoire de l'éducation, l'auteur nous présente d'abord la situation de l'éducation au début du xixe siècle (surtout en France). Par la suite, elle analyse les changements dans l'éducation, jusqu'à l'entre deux guerres, en tenant compte des transformations des divers pôles de l'activité économique, activité qui, avec l'industrialisation, requiert un personnel de plus en plus instruit, cependant pas "trop", sinon il pourrait mettre en question le système. Bien que l'auteur constate une amélioration du niveau d'instruction, elle remarque que chaque classe sociale a son propre réseau et qu'il y a peu de communication de l'un à l'autre. Enfin l'auteur analyse la situation de l'éducation dans les colonies où se révèle tout particulièrement le rapport étroit entre le fait éducatif, l'économie et la politique. Très diversifiées les politiques scolaires coloniales n'ont peut-être eu en commun que de dénier toute valeur aux coutumes et croyances locales.
Tous droits réservés @ C Les Presses de l'Université de Montréal, 1980

Ce document est protégé par la loi sur le droit d'auteur. L'utilisation des services d'Érudit (y compris la reproduction) est assujettie à sa politique d'utilisation que vous pouvez consulter en ligne.

https://apropos.erudit.org/fr/usagers/politique-dutilisation/ 


\section{Travail, rapports sociaux éducation en Europe Esquisse historique de la chute de l'Empire à l'entre deux-guerres}

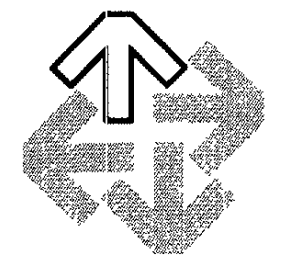

VIVIANE ISAMBERT-JAMATI

En 1815 toute une partie de l'Europe possédait, pour la première fois, les bases d'un système scolaire et non plus seulement une constellation d'établissements résultant d'initiatives non coordonnées. On a insisté sur les excès de la centralisation de ce système; on aurait dû marquer aussi sa diffusion sur le territoire, Napoléon ayant conservé (et géographiquement étendu) certains traits des réalisations scolaires jacobines. Marquer aussi son caractère de classe : école bourgeoise fort bien mise en place, avec un contingent étroit mais régulier de bourses permettant le drainage de quelques éléments nouveaux à chaque génération; école populaire, rurale et urbaine, instituée mais sans grands frais et à peine contrôlée.

Les transformations de ce système au cours du xixe siècle, puis jusqu'à la Seconde Guerre mondiale, sont trop souvent ignorées des sociologues qui s'intéressent à son état actuel et tendent à supposer de simples «traditions». Or depuis une dizaine d'années sont apparues d'excellentes recherches d'histoire de l'éducation. Les unes permettent de définir la clientèle d'élection de telle institution scolaire entre telles et telles dates, tout en identifiant les transformations qu'elles prétendaient opérer chez leurs élèves. D'autres, retournant la perspective, portent plutôt sur l'éducation reçue, les niveaux et les types de savoirs possédés par ceux qui, à tel moment, contribuaient de telle façon au fonctionnement économique et social. Ainsi est-il devenu possible, très au-delà 
des définitions officielles, de situer les divers types d'éducation dans une structure, chaque élément étant compris grâce à sa place par rapport aux autres.

Enfin on ne saurait comprendre aucun aspect de la vie économique et sociale dans l'Europe de cette période sans introduire le rapport aux colonies. Les faits éducatifs ne font pas exception et l'analyse là aussi est facilitée par quelques bonnes études historiques.

A L'Europe du premier tiers du XIXe siècle est évidemment avant tout une Europe paysanne. Des paysans que les guerres ont parfois sortis de leur terroir, brassés avec bien d'autres, mais dont la vie n'est guère comparable avec celle des citadins, les possibilités de communication étant encore très sommaires. Angleterre mise à part, au moins les trois quarts de la population européenne habitent en zone rurale et plus de la moitié des actifs travaille la terre. Or même si dans toute l'Europe occidentale, par rapport auX XVIIe et XVIIIe siècles, les charges féodales et les impôts se sont allégés pour les paysans, si les famines sont en régression et si la tendance à une centralisation administrative et policière augmente la sécurité quotidienne, les techniques agricoles elles, n'ont pas subi de transformation: le travail reste très dur et peu productif; il y faut beaucoup de bras. Bien entendu les modes de faire valoir, les formes de travail, les conditions d'existence de la paysannerie connaissent d'énormes variations géographiques, et de même que cette diversité aide à comprendre les variations des phénomènes démographiques (mortalité, âge de mariage, nombre d'enfants), de même il faudrait la connaître pour comprendre les variations du degré d'instruction.

L'Angleterre faisait, il est vrai, du point de vue législatif une sorte d'impasse sur son agriculture... et sur ses paysans. Même dans le dernier tiers du $\mathrm{XIX}^{\mathrm{e}}$ siècle, lorsque l'obligation scolaire touche l'ensemble des populations non agricoles, les enfants «occupés à l'agriculture» peuvent en être dispensés par simple lettre des parents aux autorités locales. Réalisme certes, car ces parents n'auraient pas respecté la loi; mais cette mesure aurait été fort imprévoyante si, de fait, le travail de la terre avait demandé des hommes et des femmes instruits.

Pourtant, on n'aborde pas des campagnes analphabètes lorsqu'on s'attache à l'état de l'instruction paysanne dans l'Europe de 1815: c'est avant cela que les scores d'alphabétisation rurale - du moins pour les hommes - ont décollé dans les pays protestants ou dans la France du Nord et de l'Est. Soixante à soixante-dix pour cent d'hommes sachant signer leur nom n'est pas un chiffre rare dans les cantons ruraux de ces régions françaises au moment de la révolution. La première moitié du XIXe elle, voit augmenter l'instruction masculine dans les régions rurales de la bordure méditerranéenne, et celle des femmes (à un niveau beaucoup plus bas) un peu partout. Mais dès que le mode de faire valoir entraîne le recours à d'assez nombreux journaliers, un écart se creuse : les propriétaires (qui paient de leur personne certes, mais qui aussi s'intègrent progressivement à l'économie de marché, font leurs comptes et parfois amélio- 
rent leurs techniques) auront presque tous une instruction minimum, alors que les journaliers seront nombreux à n'en pas avoir du tout ${ }^{1}$.

De plus, il semble que dans bien des cas (mais sans uniformité sur un même territoire national), l'incidence du rang dans la fratrie ait été assez grande sur la scolarité des enfants de paysans au XIXe siècle. L'instruction était la revanche des cadets : l'aîné restait traditionnellement à la terre; il fallait donc qu'un jour ou l'autre il devienne responsable et si possible propriétaire de l'exploitation. Mais comme souvent la tradition et la loi (égalité des descendants directs devant l'héritage) ne convergeaient pas, il fallait que les plus jeunes puissent s'en aller, de préférence sans demander leur part. L'entrée dans le clergé - qui passait d'ailleurs par une certaine instruction - avait largement fonctionné ainsi, mais d'autres états jouaient de plus en plus un rôle analogue : celui d'instituteur, bien sûr, mais aussi celui d'employé, de petit fonctionnaire de toutes sortes qüi, à la fin du siècle, se recrutaient souvent directement dans les familles paysannes. Ce qui bien entendu n'excluait pas le départ très fréquent de la terre familiale vers le salariat industriel, départ dans lequel le rôle de l'instruction était beaucoup moins grand.

B Qu'en est-il dans le même temps de l'instruction des ouvriers? La fin du Xvine et le début du XIXe, parallèlement à tant de bouleversements politiques, sont prodigieusement féconds en inventions techniques qui, rapidement associées à des formes nouvelles de propriété des moyens de production, vont permettre un énorme développement de l'industrie. La première caractéristique de l'enfance ouvrière - ou du moins d'une grande fraction de cette enfance - au milieu du siècle en France, en Angleterre mais aussi dans d'autres pays qui s'industrialisent à grand pas, c'est d'être exploitée très tôt. Pourtant même dans une esquisse aussi rapide, il faut de ce point de vue distinguer entre les formes d'industrialisation. Trois formes de production, en proportion inégale au long du siècle bien entendu, rendent à peu près compte de cette diversité :

- De petites fabriques quasi-rurales, généralement de type manufacturier, emploient une main-d'œuvre qui vit encore partiellement de l'agriculture; si non seulement des hommes, mais des femmes y travaillent très jeunes, on a peu recours aux enfants à proprement parler; c'est aux travaux des champs, de façon saisonnière, qu'ils contribuent.

- Des usines de régions industrielles (souvent mono-industrielles, ce qui entraîne une dépendance totale du salariat) fonctionnent avec une division tech-

1. Aux États-Unis, l'écart était plus grand encore. Aux deux siècles prêcédents des terres immenses s'étaient révélées disponibles à mesure de la conquête, sans aucune tradition de culture par parcelles, et avec un réservoir d'esclaves inépuisable: tout avait été réuni pour une exploitation extensive. Une fois l'esclavage aboli, le recours massif aux travailleurs de couleur continue; pour eux, à la fin du xixe siècle encore, pratiquement aucune scolarité. Mais chez les «farmers » on ne restait pas ignorant, même si l'on méprisait fort les raffinements intellectuels. Précisément parce que l'exploitation était extensive, on pouvait diviser très radicalement le travail; l'expérimentation ne posait pas de problème de rendement immédiat, et elle fut très précoce en Amérique; l'amélioration des outils, puis des machines, devenait indispensable, dès le moment où elle était technologiquement possible. C'est pourquoi on voit apparaître dès les années 80 un assez grand nombre de techniciens de l'agriculture; pour utiliser leurs services, les familles de propriétaires devaient pouvoir les comprendre, donc avoir quelques bases scientifiques. Ainsi dans les zones rurales on trouve une scolarité forte dans la population blanche mais presque nulle dans la population de couleur. 
nique du travail très accentuée, et assez peu d'appel à une compétence éduquée: les machines sont «servies» par les ouvriers. C'est là qu'on emploie les enfants très tôt, dans des conditions exténuantes et d'ailleurs avec une mortalité incroyable. On leur confie des tâches très élémentaires, rendues possibles par les progrès de la technologie; et comme les salaires ne permettent pas de faire vivre une famille, les parents sont contraints de les faire travailler ainsi. Il va de soi qu'en pareil cas la fréquentation d'une école est impossible. Si jamais un rapide apprentissage de la lecture et de l'écriture a eu lieu avant l'entrée à l'usine, tout sera bientôt oublié dans de telles conditions de vie. En Angleterre dans les années 1840, un règlement obligeait bien les employeurs à réunir les enfants-ouvriers pendant un moment chaque jour sous la conduite d'un maître qui leur donnerait une instruction minimale; mais, raconte Marx, lorsque par hasard cette règle était appliquée, le «maître» se révélait souvent lui-même analphabète...

- Des ateliers semi-mécanisés, enfin, sont surtout développés dans des villes de moyenne importance, ainsi qu'à la périphérie des très grandes villes à fonctions multiples. La proportion de travailleurs de métier y est forte, et l'embauche porte en partie sur des apprentis qui, tout en contribuant à la production, s'initient à la qualification. À ceux-là il faut des connaissances scolaires «de base» (ce terme n'étant bien évidemment pas univoque) car ils rencontrent déjà des problèmes de cotes, de rapports proportionnels, de consignes de travail présentées par écrit, etc.

C'est pourquoi dans les deux premiers tiers du siècle les intérêts des industriels en matière d'instruction des futurs ouvriers ne présentent aucune uniformité. Qu'en est-il des ouvriers eux-mêmes? Certains des plus avancés, de ceux qu'on appellerait de nos jours des «militants» ont laissé sur l'éducation des écrits saisissants; mais la distance entre leurs attitudes et celles du grand nombre est impossible à estimer. Quant à la diversité des formes industrielles qui viennent d'être rappelées, elle entraînait évidemment chez eux des positions très diverses, qui ne peuvent être analysées ici.

Encore au milieu du siècle, habiter une ville de grosse industrie donne de fortes chances d'être analphabète. Les chiffres disponibles sur l'alphabétisation permettent rarement de distinguer, au sein de telle ou telle aire, les catégories professionnelles, mais si l'on connaît la composition sociale des agglomérations, ou même des quartiers, des regroupements peuvent se faire. Par exemple en France Le Creusot, ville minière et métallurgique, a $54 \%$ d'analphabètes en 1845, alors qu'à Autun, ville très voisine mais non industrielle, on n'en compte que $25 \%$. Dans les quartiers ouvriers des capitales européennes, ou dans de très grandes villes multifonctionnelles, le phénomène est différent. Non seulement les ouvriers subissent une certaine contagion des comportements d'autres couches sociales, mais les travaux des industries de transformation, et plus encore ceux des industries de luxe, exigent une qualification. C'est surtout dans ces villes que s'étaient développées, en Angleterre dès le début du siècle, un peu plus tard en France, les écoles mutuelles. Comportant une organisation quasi-industrielle, elles éduquaient à la régularité, mais leur rendement en jeunes gens instruits était assez faible : on les quittait souvent tôt. À Paris à la veille de la Commune, bien des témoignages font état d'une scolarisation populaire très courte et pour certains, inexistante. Quant à l'éduca- 
tion proprement professionnelle, sous une forme scolaire, elle était des plus rares.

Au même moment, dans les sociétés mutualistes, à propos des expositions, dans les journaux ouvriers, le droit à l'éducation pour le peuple était très souvent revendiqué par les travailleurs. D'où en France les initiatives de la Commune en matière d'éducation «intégrale » (c'est-à-dire prenant l'homme dans son entier, et non pas seulement soit sa sensibilité esthétique, soit sa capacité de travail), mais aussi d'éducation «pour tous».

Dans le dernier quart du siècle enfin, l'obligation scolaire pour les masses - à condition qu'elle n'aille pas plus loin qu'une instruction élémentaire n'a plus guère rencontré d'opposition. Les lignes précédentes le laissaient prévoir : c'est un cas particulièrement net de mesure répondant à tant de motifs divers, et même d'intérêts par ailleurs opposés, qu'elle est venue comme un fruit mûr, seules les modalités (notamment religieuses en France) ayant provoqué de réels conflits. Même pour les propriétaires de grandes fabriques, remplacer l'usage très précoce de la force de travail par une courte phase de scolarisation, permettant d'économiser les capacités physiques des enfants de la classe ouvrière en vue d'un usage ultérieur, s'avérait utile. Or il fallait pour cela une obligation légale, sous peine de voir les concurrents se comporter, eux, autrement et donc produire à plus bas prix. Une autre partie du patronat et des cadres de l'industrie, on l'a vu, avait un besoin direct d'une école qui munisse ses futurs salariés non seulement de la lecture et de l'écriture, mais (les techniques acquérant un type de complexité que je ne puis analyser ici), d'une certaine posture intellectuelle rationnelle. C'est en revanche seulement pour atténuer le fameux danger constitué par les classes laborieuses que les élites traditionnelles (hobereaux, homme de loi, haut clergé) ont fini par tolérer et parfois même demander une instruction dûment contrôlée des enfants du peuple; mais pour l'ordre social des rudiments suffisent : ces notables comptaient, pour moraliser, sur les apprentissages élémentaires, qui ne peuvent se faire sans un minimum de discipline. Les classes moyennes d'encadrement, nouvelles élites républicaines, souhaitaient quant à elles s'acquérir une clientèle politique : adeptes des lumières, elles comptaient sur les masses «libérées» par l'instruction pour appuyer leur propre émergence. Quant aux ouvriers eux-mêmes, qui avaient peu à peu obtenu des salaires permettant à la rigueur de se passer pendant quelques années du salaire de leurs enfants, la scolarisation pouvait répondre chez eux à plusieurs visées; voir leurs enfants échapper aux tâches les plus pénibles des usines, soit grâce à une qualification ouvrière, soit parce qu'ils deviendraient employés ou petits cadres, nè pouvait leur être indifférent; mais aussi, de plus en plus nombreux ils estimaient que, chez ceux-même qui font un travail d'exécution, l'instruction augmente les possibilités de discuter, de s'organiser, bref, de se défendre...

C L'éducation des jeunes privilégiés au cours du xıxe siècle est mieux connue que celle des autres couches sociales. Ayant constamment été un phénomène de classe bien caractérisé, elle présente encore plus de parenté d'un pays d'Europe à un autre, d'autant plus qu'elle est largement tributaire d'un réseau institué aux $\mathrm{XVI}^{\mathrm{e}}$ et $\mathrm{XVII}{ }^{\mathrm{e}}$ siècles par les Jésuites dans tout le monde occidental. 
La bourgeoisie, dès ses origines, professe une sorte de culte de «la réussite due au travail», qui à la fois reflète les difficultés éprouvées pour mener ses entreprises et masque le profit tiré du travail épuisant des salariés... Aussi aurait-elle pu vouloir que ses enfants soient tôt initiés aux problèmes de production, à la technique, à la tenue des livres. Mais si le mode de vie, le rapport au travail et à l'argent, étaient en rupture avec ceux de l'aristocratie, il fallait conserver certains signes distinctifs de cette classe pour être reconnu comme ses héritiers politiques légitimes. C'est un des sens que prit l'enseignement donné dans les lycées. On n'est plus (et on ne veut plus être) défini par l'oisiveté à vie, mais du moins offre-t-on à ses enfants de longues années dégagées de toute nécessité, des années de loisir pour qu'ils se cultivent et ornent leur esprit de sagesse, de vérité et de beauté. Grâce à cet hommage à des valeurs qu'elle nomme «spirituelles» et «éternelles», la bourgeoisie justifie le statut politique qui, dans les pays occidentaux, renforce sa réussite matérielle. Qu'il soit dispensé par des religieux ou des laiques, l'enseignement secondaire des trois premiers quarts du $\mathrm{XIX}^{\mathfrak{e}}$ siècle ne différait guère : les disciplines classiques, en particulier les langues anciennes, formaient l'activité essentielle.

À côté des fils d'une bourgeoisie libérale déjà ancienne, en particulier d'hommes de loi, de médecins, d'administrateurs de rang élevé, furent instruits de cette façon un nombre croissant de fils d'industriels (qu'ils soient propriétaires ou collaborateurs immédiats des propriétaires, car la dénomination $d$ ' «ingénieur» ne servait pas encore couramment) et de gros commerçants. À mesure qu'elle se renforçait, la bourgeoisie du milieu du siècle prévoyait ainsi pour sa jeunesse non seulement une fusion culturelle, mais l'occasion d'alliances de toutes sortes (en particulier de mariages : les aristocrates s'étaient mariés d'après le quartier de noblesse; les bourgeois épousaient les sœurs de leurs condisciples).

Fusion, niveau, dira Goblot, mais aussi barrière : en Allemagne, en France, en Angleterre lorsque se créent avec plus ou moins d'ampleur des écoles prolongeant l'enseignement primaire pour la toute petite bourgeoisie industrieuse, elles sont toujours définies comme profondément distinctes des établissements secondaires de culture générale. La continuité serait «contre nature». Quant au contenu de ses études, même si ses programmes subissent des variations, l'enseignement de la bourgeoisie se veut purement classique jusqu'en 1880 . Et surtout il se veut très minoritaire, réservé à une élite, les textes qui le définissent présentant constamment une ambiguïté entre la référence à une pure et simple prédestination sociale (la faiblesse des effectifs reflétant alors simplement la faiblesse numérique de la couche concernée) et la référence à un mérite intellectuel, particulièrement rare. En vérité ce public représente entre 1 et $4 \%$ des jeunes gens selon le pays et la date considérés, avec une croissance très lente, qui traduit numériquement la barrière.

Le début du xxe siècle, plus particulièrement à l'occasion de la Première Guerre mondiale, connaît d'énormes bouleversements économiques et sociaux. 
Les systèmes éducatifs n'ont pas cessé d'être en étroite correspondance avec les systèmes des classes sociales; mais les savoirs et les dispositions ethicointellectuelles attendus de leurs membres respectifs ont changé.

A Dans les campagnes, l'isolement a presque disparu. Ce n'est plus seulement pour les enfants destinés à la mobilité géographique et sociale qu'on demande de l'instruction, mais aussi pour les futurs cultivateurs, en cours de régression numérique il est vrai; sauf dans quelques régions, l'obligation scolaire, lorsqu'elle existe, est de plus en plus respectée sur toute sa durée. $\mathrm{Au}$ cours des années 30 on commence même à voir des garçons prolonger l'enseignement par une brève formation à l'agriculture, et des filles recevoir quelques cours ménagers. En France en tous cas ces initiatives, souvent cléricales, ont tendu pour une part à maintenir sur ces jeunes une emprise idéologique traditionnelle, pour éviter que le séjour à l'école laïque renforcé par la circulation des idées, n'aboutisse à un effritement religieux; mais elles eurent aussi des objectifs économiquement modernisateurs: faire adopter de nouvelles techniques agricoles et inciter à la consommation de nombreux produits manufacturés.

B Le mouvement de scolarisation des enfants d'ouvriers s'est lui aussi poursuivi rapidement dans les dernières années du XIXe, puis, plus lentement, dans la première moitié $\mathrm{du} \mathrm{xx}^{\mathrm{e}}$. L'obligation d'envoyer les enfants à l'école s'était accompagnée de l'interdiction, pour les employeurs, de les faire travailler. Dans les villes, l'une et l'autre furent d'autant plus rapidement respectées que le fait avait déjà largement devancé la loi. Il est vrai que dans 1'industrie c'est le travail spécialisé, morcelé et répétitif qui prend de plus en plus d'importance; il est même à la source de l'augmentation continue des effectifs de l'industrie. Ce travail n'exige pratiquement pas de connaissances techniques, mais il suppose un minimum de repérages et de maniements symboliques qu'une instruction de base favorise. Quant aux emplois industriels qualifiés, ils tendent assez rapidement à se transformer : l'expérience des matériaux, l'habileté manuelle lentement acquise sur l'établi même, régressent; on voit se développer des tâches plus indirectes, en particulier des commandes d'opérations qui supposent une saisie (au moins partielle) du système technique en jeu. D'où la tendance à développer une formation professionnelle accompagnée d'un certain prolongement d'instruction. Selon le pays, il s'agit surtout de cours professionnels à temps partiel, la majeure partie du temps se déroulant à l'atelier, ou d'établissements scolaires techniques, eux-mêmes dotés d'un équipement en machines. Selon le cas aussi le patronat ou les pouvoirs publics prennent les initiatives. Mais quelle que soit la situation, la visée en élaborant les programmes est double: munir ces jeunes gens de ce qu'il faut pour qu'ils deviennent des ouvriers qualifiés efficaces, et pourtant éviter de les instruire «trop», de peur que devenus critiques (et capables d'autres tâches), ils ne cessent d'accepter leur situation d'ouvriers.

Parallèlement à la concentration industrielle, dans la première moitié de ce siècle, se développe le tertiaire : bureaux, commerces, services. Celui-ci va donc absorber de plus en plus d'employés et l'on voit naitre des formations qui lui sont propres. Tout en étant spécifiques cependant, ces formations adop- 
tent une structure calquée sur celle des écoles industrielles : il s'agit de la même population (côté féminin très souvent) et les précautions pour que les acquisitions ne dépassent pas le nécessaire sont les mêmes... L'enseignement pour les enfants d'ouvriers, futurs salariés de rang modeste, qu'ils restent ou non dans l'industrie, présente donc dès ce moment deux faces complémentaires, comme c'est le cas de nos jours.

C La scolarisation s'est donc allongée pour des milieux que l'école ne touchait jusqu'alors que très brièvement. Mais les milieux intellectuels, même avancés, continuent à accepter la prédestination scolaire : l'idée selon laquelle à partir d'une éducation de base commune, tous les jeunes d'une génération devraient pouvoir, selon leur mérite, recevoir une formation «courte» ou une formation «longue» n'est pas encore monnaie courante dans les années 20. Quant aux déclarations officielles, quel que soit le pays, aucune ne formule encore de prétention à «l'égalité des chances». Si, dans beaucoup de cas, les effectifs du secondaire se sont un peu élevés juste avant la seconde guerre mondiale, cela signifiait l'intégration de quelques individus issus du peuple, mais non un changement de fonction. L'ordre de grandeur de cette population secondarisée est resté très limité, traduisant la quasi-exclusivité dont jouissaient les jeunes de famille privilégiée, et donc la différenciation sociale profonde et presque explicite des systèmes scolaires.

Les programmes de l'enseignement secondaire général sont loin, eux, d'être restés immuables, leur adaptation étant réclamée par des exigences externes. En effet des professions comme la médecine, par exemple, faisaient appel depuis le début du siècle à une scientificité croissante. Mais surtout pour faire fonctionner dans divers secteurs l'économie capitaliste, on ne pouvait plus se contenter de références à l'antiquité et de raffinements esthétiques. Les marchés s'étaient internationalisés, rendant indispensable la possession des langues vivantes pour faire du commerce à un haut niveau. Et surtout l'industrialisation se poursuivait, mettant en œuvre des connaissances de plus en plus importantes et toujours renouvelées. Aussi la proportion de jeunes gens se dirigeant après le secondaire «général» vers des études d'ingénieur, ou en tous cas de haute technicité, a-t-elle, au cours de cette période, augmenté d'année en année.

De plus cette orientation de l'activité chez une assez large fraction de l'élite sociale a progressivement influencé les composantes de la «culture générale». Beaucoup plus rapidement dans les pays anglo-saxons que dans les pays latins, et dans tous les cas plus nettement pour les hommes que pour les femmes, des savoirs (ou du moins des aperçus) scientifiques se sont intégrés pendant cette première moitié du siècle aux signes de reconnaissance de la classe cultivée. Partout, enfin, avec l'élargissement des possibilités de déplacement, la pratique des langues étrangères s'est mise à figurer elle aussi dans la culture de l'honnête homme.

Pour toutes ces raisons, les sciences et les langues vivantes ont pris de l'importance dans les établissements secondaires, soit pour une partie des élèves, avec une spécialisation des sections, soit pour tous. Dans les pays de tradition classique très ancrée, cependant, le corps enseignant et une partie du public cultivé ont persisté à proclamer le caractère gratuit de tous les program- 
mes. Il ne fallait à aucun prix que l'enseignement professionnel récemment développé puisse apparaître comme une variante de l'enseignement secondaire. Durkheim, au début du siècle, prend cette distance vigoureusement à son compte : «il importe au plus haut point, écrit-il, de les distinguer». Tout au plus ces disciplines nouvelles peuvent-elles constituer une "gymnastique intellectuelle. Mais quelle que soit l'idéologie qui les a accompagnées, leur extension tendait cependant à transformer l'enseignement secondaire.

Tel est donc, dans les pays d'Europe occidentale, l'état des systèmes scolaires au moment de la seconde guerre mondiale : il n'y a plus d'analphabètes, la durée de la scolarité s'est pour tous allongée, mais chaque classe sociale a bien son réseau, avec peu de communication de l'un à l'autre.

\section{III}

A Au cours de cette période les pays d'Europe ont donc connu d'énormes transformations de technologie et de modes de vie. Transformations dont la physionomie est certes profondément différente selon la place occupée dans les rapports sociaux, mais qui touchent néanmoins la population entière. Là, en effet, les progrès massifs dans la maîtrise de la nature, même s'ils se traduisent principalement en profits pour une minorité, se traduisent aussi (et de façon nécessaire au système) en apparition constante de nouveaux besoins pour la grande majorité. Dans des continents entiers, le système économico-politique a été, et est encore autre. Restés pratiquement en dehors de ces bouleversements jusqu'aux environs de 1870, ils ont été à partir de là l'objet de transformations économiques partielles, caractérisées par la surexploitation et la désarticulation, et entièrement ordonnées aux intérêts des pays industriels cherchant leur propre expansion.

Les institutions éducatives n'ont évidemment jamais représenté l'essentiel de l'appareil colonial de ces années. Elles y jouent pourtant un rôle, alors que la question ne se posait nullement dans les formes antérieures de colonisation. Et les rapports de l'éducatif avec l'économique et le politique furent alors si manifestes qu'ils ont parfois servi aux analystes de révélateurs particulièrement puissants d'une de ses significations sociales majeures.

On croit parfois pouvoir opposer les mesures relatives à l'enfance (ou à certains enfants) introduites par la politique coloniale de telle ou telle période à une «éducation traditionnelle» qui, finalement, aurait présenté toutes sortes de traits communs d'une région colonisée à une autre. Faut-il rappeler que cette éducation «naturelle» commune à une humanité qui serait près de ses origines est illusoire? Des sociétés isolées, ou relativement isolées, ont pour les jeunes générations des pratiques de socialisation chaque fois différentes, non seulement d'un peuple à l'autre, mais même d'une petite communauté à une autre. Même dans les cas où un système de pensée s'est répandu sur des aires très vastes, l'interprétation éducative des préceptes éthico-religieux (d'une grande généralité la plupart du temps) connaît d'énormes variations. Entre les populations islamisées du Moyen-Orient, celles du Maroc et celles du Mali, les similitudes entre les «traditions» éducatives sont fort ténues... Et la variété est encore plus 
grande lorsqu'un tel élément unificateur n'existe pas. En toute hypothèse l'éducation antérieure à l'arrivée des occidentaux ne fut jamais ni l'ensemble de coutumes barbares décrites par les missionnaires et les colons, ni l'ensemble de pratiques idylliques projetées dans le passé par certains intellectuels contemporains.

Mais la colonisation a toujours dénié toute valeur aux coutumes et aux croyances locales : c'est là peut-être le seul trait commun, car les politiques scolaires coloniales ont connu une double source de variation. Les colonisateurs, pour installer leur pouvoir et mettre en place quelques éléments de leur technologie, ont inégalement estimé la fraction de population à instruire; pour réaliser cette instruction, de plus, ils n'ont pas tous imposé de la même façon leur religion, leur culture, leur langue. Les colonisés, eux, qui attachaient une importance variable à tels et tels aspects de la socialisation des jeunes générations, ont aussi diversement résisté aux contraintes extérieures à cet égard.

B Entre les années 70 et la Première Guerre mondiale environ, la plupart des pays colonisateurs ont soit favorisé quelques écoles missionnaires, soit créé des écoles liées au gouvernement colonial, mais toujours pour une très faible fraction de la population et à un niveau très élémentaire. La préoccupation principale était de susciter une petite couche intermédiaire, qui n'aurait pu jouer son rôle sans posséder, outre un certain maniement de la langue des colons, des éléments de lecture, d'écriture et de calcul. Grâce à cela, les futurs intermédiaires de l'action colonisatrice devaient «être disposés à se rapprocher de nous», comme l'écrivaient par exemple les textes officiels français de 1898. Fréquenter ces écoles était d'ailleurs présenté comme un rare privilège, réservé aux «fils de chefs» - ce qui fut en réalité suivi des substitutions que l'on sait. Mais dès ce moment les effectifs ainsi touchés furent très différents selon le type d'exploitation du territoire. Il fallait une couche d'alphabétisés plus importante lorsque les Européens étaient eux-mêmes nombreux sur place, et lorsque la population devait fournir un effectif important de main-d'œuvre : l'encadrement à la base devait être assuré, sans oublier la vie quotidienne (confort et sécurité) des colons. Dans les cas de présence principalement stratégique, ou même d'exploitation très sectorielle, on ne prit la peine d'instruire que quelques dizaines $d^{\prime}$ ' « indigènes » à chaque génération.

Dans les pays où pré-existait une culture écrite et une transmission institutionnalisée de cette culture, des compromis ont souvent été tentés empiriquement; mais l'acculturation au pays exploitant, et donc la dépossession culturelle, ont toujours prédominé de beaucoup, étant donné la finalité poursuivie. En l'absence de telles traditions, la faveur accordée en scolarisant une petite fraction de la population se donnait pour plus grande encore. Ce n'était pourtant guère plus qu'une alphabétisation. Les commentaires de l'époque sont pleins de restrictions, d'appels à la prudence pour que la scolarisation reste exceptionnelle et limitée : les « indigènes » trop instruits deviendraient présomptueux, n'accepteraient plus, individuellement, la complète soumission. Au reste, prétendit à plusieurs reprises une «psychologie coloniale», ils n'étaient pas capables d'accéder aux notions difficiles: il leur fallait des notions très simples et très morales. Le risque proprement politique fut d'ailleurs souvent évoqué : rassemblant des jeunes gens qui venaient de villages dispersés, faisant acquérir 
des instruments (ne serait-ce que l'écriture) utilisables pour relier des hommes géographiquement éloignés, les écoles pouvaient devenir des foyers de subversion. Conscients de ces dangers, les administrateurs les surveillaient étroitement et, lorsque par hasard existaient des maîtres autochtones (écoles coraniques par exemple), ils étaient strictement contrôlés. Quant à la longueur de la scolarité, c'est dans cette perspective aussi, et non pas seulement pour réduire les compétences acquises, qu'on la restreignait.

C Lorsque deux ou trois générations eurent connu cette amorce d'école, les restrictions draconiennes durent se relâcher. Si peu que ce soit, l'instruction s'était malgré tout révélée rentable pour ses quelques bénéficiaires. En effet, les activités productives traditionnelles ayant décliné au profit des formes de travail dépendantes, surexploitées, mais inéluctables, une scolarité, si faible soitelle, prenait une certaine valeur économique. Aussi non seulement ceux qui avaient eux-mêmes fréquenté les premières écoles, mais aussi d'autres demandaient une scolarité moins rudimentaire pour leurs enfants. Les réponses furent diverses. La plupart du temps, plutôt que de mécontenter les «indigènes» et surtout les «indigènes évolués» sur lesquels ils s'appuyaient, les pays colonisateurs ont, vers les années 30 , concédé un certain élargissement de leurs créations d'écoles primaires. Au reste, une fois qu'elles étaient réclamées, on pouvait espérer les voir produire des effets accrus d'inculcation idéologique, et donc de consolidation du rapport colonial. Elles pouvaient, de plus, permettre d'économiser l'emploi de cadres subalternes européens qui coûtaient cher.

Pourtant cette action n'a jamais été géographiquement uniforme au sein d'un même ensemble colonial. Les créations d'écoles, en effet, ont bien souvent reflété la politique inégale d'une région à une autre: ainsi les régions côtières étaient souvent nettement mieux pourvues que les autres: non seulement on privilégiait les «zones de contact $»$, mais on divisait pour régner...

Enfin, totalement absent dans les premières décennies de la colonisation, l'enseignement destiné en Europe aux jeunes gens de la bourgeoisie (lycées, gymnases, collèges religieux largement subventionnés) est apparu dans quelques colonies autour de 1925. Il ne s'adressait en principe qu'aux enfants des colons. Pourtant ici ou là quelques fils de familles autochtones particulièrement proches de l'administration coloniale, très spécialement «amies de la métropole», y ont été admis. Pour ces happy fews, très au-dessus des simples «évolués», pas question d'adapter l'enseignement aux réalités locales, pas question non plus d'abaisser le niveau pour se mettre à leur portée: exceptions confirmant la règle, c'est à l'ensemble du banquet qu'ils étaient conviés. Une culture prestigieuse passait pour préférable à toute compétence technique. Peu importe qu'ils représentent beaucoup moins d'un sur 100000 adolescents à chaque génération : ils devenaient symboles de l'assimilation impossible et pourtant prodigieusement réalisée - objets supposés d'une admiration rejaillissant sur le pays colonisateur. Faut-il insister sur l'ambivalence de ce statut, tôt ou tard perçu par beaucoup comme une trahison... Mais notre propos s'arrête en 1945: les empires semblent tenir encore. 
Sachant mieux comment se sont transformés les savoirs requis aux divers pôles de l'activité économique, on perçoit plus clairement pourquoi les systèmes éducatifs ont changé au cours de cette période. Mais pas plus que sur un autre terrain il n'y a de formule universelle pour saisir le sens de l'histoire éducative : faire, comme ici, l'impasse sur l'histoire culturelle, aussi bien scientifique qu'esthétique, c'est supposer un peu mécaniquement une rationalité économique de la politique scolaire. Là aussi, l'apport récent des historiens pourrait servir à une réinterprétation plus élaborée, qui unirait les deux aspects, et éclairerait davantage les caractères de l'éducation contemporaine.

\section{BIBLIOGRAPHIE INDICATIVE}

ARCHER, M.S., Social Origins of Educational Systems, Londres, Sage, 1979.

BISHOP, A.S., The Rise of a Central Authority for English Education, Cambridge, Cambridge University Press, 1971.

BOON, H., Enseignement primaire et alphabétisation dans l'agglomération bruxelloise de 1830 à 1879, Louvain, Nauwelaerts, 1969.

CHEVALIER, L., Classes laborieuses et classes dangereuses à Paris, Paris, Plon, 1958.

CHEVALLIER, P., B. GROSPERRIN et J. MAILLET, l'Enseignement français de la Révolution à nos jours, Paris, Mouton, 1968.

COLONNA, F., Instituteurs algériens, 1833-1939, Paris, Fondation nationale de science politique, 1975.

DUBY, G. et R. MANDROU, Histoire de la civilisation française, Paris, Colin, 1977.

DUVEAU, G., la Pensée ouvrière sur l'éducation pendant la Seconde république et le second Empire, Toulouse, Domat-Montchrestien, 1948.

FURET, F. et J. OZOUF, Lire et écrire, l'alphabétisation des Français de Calvin à Jules Ferry, Paris, Minuit, 1978.

ISAMBERT-JAMATI, V., Crises de la société, crises de l'enseignement, Paris, P.U.F., 1970.

JARMAN, T.L., Landmarks in the History of Education, Londres, J. Murray, 1963.

KITA, K., la Politique scolaire de la Belgique au Congo, 1880-1962, Kinshasa, 1976.

LUNDGREEN, P., «Industrialization and the Educational Formation of Manpower in Germany», Journal of Social History, 9, 1975, p. 64-80.

MURPHY, J., Church, State and School in Britain, 1800-1970, Londres, Routledge, 1971.

PARIAS, G. (éd.), Histoire générale du travail, Paris, Nouvelle librairie de France, 1960.

SIMON, B., Education and the Labor Mouvement, 1870-1920, Londres, 1965.

TANGUY, L., "L'État et l'école, l'école privée en France», Revue française de sociologie, 13, 1972 , p. $325-375$.

VAUGHAN, M. et M. SCOTFORD-ARCHER, Social Confict and Educational Change in England and in France, 1789-1848, Cambridge, Cambridge University Press, 1971.

\section{RÉSUMÉ}

Selon l'auteur, les transformations passées du système scolaire sont trop souvent ignorées des sociologues. Se basant sur des recherches récentes d'histoire de l'éducation, l'auteur nous présente d'abord la situation de l'éducation au début du xıxe siècle (surtout en France). Par la suite, elle analyse les changements dans l'éducation, jusqu'à l'entre deux guerres, en tenant compte des transformations des divers pôles de l'activité économique, activité qui, avec l'industrialisation, requiert un personnel de plus en plus instruit, cependant pas «trop», sinon il pourrait mettre en question le système. Bien que l'auteur constate une amélioration du niveau d'instruction, elle remarque que chaque classe sociale a son propre réseau et qu'il y a peu de communication de l'un à 
l'autre. Enfin l'auteur analyse la situation de l'éducation dans les colonies où se révèle tout particulièrement le rapport étroit entre le fait éducatif, l'économie et la politique. Très diversifiées les politiques scolaires coloniales n'ont peut-être eu en commun que de dénier toute valeur aux coutumes et croyances locales.

\section{SUMMARY}

According to the author, past transformations in the educational system have been too often passed over by Sociologists. On the basis of recent research on the history of education, the author first presents the situation of early 19th century education (mainly in France). She then analyzes the changes which have taken place in education up to the period between the two world wars, taking into account the transformations of the various poles of economic activity. Due to industrialization, an ever more educated workforce is required, not 'too' educated however, so as not to challenge the system itself. Even though the author observes an improvement in the level of instruction, she notes that each social class has its own network, with very little communication existing between them. Lastly, the author analyzes the situation of education in the colonies, where the relationship between education, economy and politics is particularly evident. Colonial educational policies vary considerably, having in common perhaps no more than the denial of any value to local customs and beliefs.

\section{RESUMEN}

Según el autor las transformaciones pasadas del sistema escolar son muy a menudo ignoradas por los sociólogos. Apoyándose en las investigaciones recientes de la historia de la educación, el autor trata la situación de la educación al comienzo del siglo diecinueve (sobretodo en Francia). Seguidamente analiza los cambios en la educación, hasta el período entre las dos guerras, teniendo en cuenta las transformaciones de los diversos polos de la actividad económica, actividad que a causa de la industrialización requiere un personal cada vez más instruído. El autor constata una mejora en el aivel de instrucción, sin embargo muestra que cada clase social tiene su propia red de instrucción, con muy poca comunicación entre una y otra. Finalmente el autor analiza la educación en las colonias, es allí donde se manifiesta de una manera muy particular la relación íntima entre el hecho educativo, la economía y la política. Las políticas escolares coloniales, muy diversas, no tienen tal vez en común nada más que el hecho de negar todo valor a las costumbres y creencias locales. 